\title{
The Role of the Spleen in
}

\section{Experimental Pneumococcal Bacteremia}

\author{
Eric J. Brown, Stephen W. Hosea, and Michael M. Frank, Laboratory of Clinical \\ Investigation, National Institute of Allergy and Infectious Diseases, National \\ Institutes of Health, Bethesda, Maryland 20205
}

\begin{abstract}
A B S T R A C T The importance of the spleen in host defense against pneumococcal bacteremia has been suggested by a number of experimental models as well as the occurrence of the syndrome of overwhelming pneumococcal sepsis in asplenic individuals. We studied the mechanism of splenic protection against pneumococcal bacteremia using a guinea pig model. Rates of removal of pneumococci from the blood stream in normal and splenectomized guinea pigs were compared with the extent of hepatic and splenic sequestration of radiolabeled organisms for three different types of pneumococci. A relationship was found between the virulence of a pneumococcus for normal guinea pigs, the extent to which it is cleared by the spleen, and the magnitude of the defect in blood stream sterilization induced by splenectomy. The spleen plays an increasingly important role in the clearance of progressively more virulent organisms, for which hepatic clearance cannot compensate. Thus, the division between hepatic and splenic clearance of bacteremia is a key determinant of the outcome of experimental pneumococcal infection.
\end{abstract}

\section{INTRODUCTION}

The importance of the splanchnic reticuloendothelial system (RES) ${ }^{1}$ in the clearance of bacteremia in both experimental animals (1-3) and humans (4) is well recognized. Moreover, it is known that different bacteria distribute differently within the RES when they are cleared (5), that opsonic requirements for RES clearance vary with the bacterial species investigated

Presented in part on 10-12 May 1980 at the 93rd Annual Meeting of the American Federation for Clinical Research in Washington, D. C.

Received for publication 15 September 1980 and in revised form 26 November 1980.

${ }^{1}$ Abbreviations used in this paper: CVF, cobra venom factor; CVF-GP, complement-depleted guinea pigs; nl-gp, normal guinea pig; PBS, phosphate-buffered saline; Pn, pneumococci; RES, reticuloendothelial system; rough $\mathrm{Pn}$, R36a; spx-gp, splenectomized guinea pig; Ty 7, type 7 Pn; Ty 12, type $12 \mathrm{Pn}$.
(6), and that the amount and type of opsonin available markedly affect the distribution of cleared organisms in the RES. ${ }^{2}$ However, the correlation between organ localization of cleared bacteria and bacterial virulence has never been systematically investigated.

The critical importance of the spleen in host defense against pneumococcal bacteremia is suggested by the occurrence of overwhelming pneumococcal sepsis in patients who are anatomically or functionally asplenic (7-9). Experimental models of pneumococcal infection have also demonstrated increased mortality and prolonged bacteremia in asplenic animals $(10-13)$. Data accumulated in humans suggest that not all pneumococci (Pn) are equally virulent for asplenic hosts (14). In the experiments reported here, we investigated the extent of pneumococcal localization in the various RES organs after experimental pneumococcal bacteremia in guinea pigs in order to understand its relationship to the virulence of the Pn. To explore this relationship further, we also investigated the blood stream clearance and lethality of these $\mathrm{Pn}$ in splenectomized guinea pigs (spx-gp).

\section{METHODS}

Pneumococci. Type 7 (Ty 7) (ATCC 6307), type 12 (Ty 12) (ATCC 6312), and R36a (rough) (ATCC 27336) Pn were obtained in lyophilized form (American Type Culture Collection, Rockville, Md.) and reconstituted in trypticase soy broth containing 5\% defibrinated sheep's blood. They were maintained on trypticase soy agar plates containing 5\% sheep's blood and Ty 7 and Ty 12 were passed in white mice monthly to maintain virulence.

Radiolabeled pneumococci. Pn were radiolabeled with ${ }^{59} \mathrm{FeCl}$ in a completely defined artificial medium (15) as described. ${ }^{3}$ Briefly, log phase Pn were grown in $40 \mathrm{ml}$ of the

\footnotetext{
${ }^{2}$ Brown, E. J., S. W. Hosea, and M. M. Frank. The role of complement in the localization of pneumococci in the splanchnic RES during experimental bacteremia. Submitted for publication.

${ }^{3}$ Brown, E. J., S. W. Hosea, and M. M. Frank. Localization and clearance of radiolabeled pneumococci in experimental bacteremia as a function of the bacterial growth cycle. Submitted for publication.
} 
medium containing $40 \mu \mathrm{Ci}$ of ${ }^{59} \mathrm{FeCl}_{3}(1 \mathrm{mCi} / \mathrm{mg}$, Amersham Corp., Arlington Heights, Ill.) for $90 \mathrm{~min}$, washed twice in ice-cold phosphate-buffered saline (PBS), and used within $1 \mathrm{~h}$.

Guinea pigs. Normal 300-400-g female guinea pigs (nl-gp) were obtained from colonies maintained by the National Institutes of Health. Splenectomy was performed on some guinea pigs via a left lateral incision under ethyl carbamate anesthesia. These guinea pigs were used in experiments 2 wk-5 mo after splenectomy. Sham operated controls, in which the peritoneum was opened and then closed, were shown to have identical clearance to nonoperated guinea pigs.

Clearance studies. The rate of removal of an intravenously injected dose of $10^{8} \mathrm{Pn}$ was determined by serial bleedings from the retro-orbital plexus at prescribed times after injection. The sample was taken into a heparinized plastic tube and a measured volume of this blood was removed for quantitative culturing. Colony-forming units were counted by culturing serial dilutions of the blood sample in duplicate on 5\% sheep's blood agar plates.

Localization studies. $45 \mathrm{~min}$ after the intravenous injection of $10^{8}$ radiolabeled $\mathrm{Pn}$, guinea pigs were killed and radioisotope in the liver and spleen was quantitated (Gamma 310 Radiation Counter, Beckman Instruments, Inc., Fullerton, Calif.). Detailed studies of clearance curves obtained after injection of log phase $\mathrm{Pn}$ into guinea pigs have previously been reported (16). In brief, there is an early exponential removal of Pn from the blood which continues for 2-4 h. In the first 5 min after injection, as many as $25 \%$ of the Pn accumulate in the pulmonary circulation but this phase of clearance has ended by $45 \mathrm{~min}$ (17). For the current experiments, 45 min was thus chosen as a time at which log phase clearance was still progressing, dilution of radiolabel by division of organisms was minimal, and radiolabel had not been released from the phagocytic cells by destruction of radiolabeled organisms through phagocyte lysosomal activity. Counts per minute in the liver and spleen of individual guinea pigs were compared by the ratio: counts per minute liver/counts per minute spleen. This ratio of hepatic to splenic radioactivity was then used as the basis for comparison of the reticuloendothelial localization for different types of Pn.

Immunization. Guinea pigs were immunized by intravenous injection of a sublethal dose of live Pn of the appropriate type 2-4 wk before an experiment. Antibody titers were measured using a modification of an enzyme-linked immunoabsorbent assay technique developed for the measurement of human anticapsular polysaccharide antibodies. ${ }^{4}$ Briefly, Ty 7 or Ty 12 polysaccharide (Merck, Sharp, \& Dohme, West Point, Pa.) was used to coat microELISA (enzyme-linked immunoabsorbent assay) plates by incubating $50 \mu \mathrm{l}$ of a 1$\mathrm{mg} / \mathrm{ml}$ solution in PBS in each well at $4^{\circ} \mathrm{C}$ overnight. After coating, plates were washed three times with PBS containing 0.1\% Tween-20 (Sigma Chemical Co., St. Louis, Mo.) (PBSTween). $50 \mu$ l of sera diluted in PBS-Tween were then added to each well, incubated for $2 \mathrm{~h}$ at $37^{\circ} \mathrm{C}$, and washed again three times with PBS-Tween. $50 \mu$ l of a 1/500 dilution of horseradish peroxidase-linked anti-guinea pig gamma globulin (N. L. Cappel Laboratories, Cochranville, Pa.) was added to each well, incubated at room temperature for $1 \mathrm{~h}$, and washed again. In the final step $200 \mu \mathrm{l}$ of $O$-phenylenediamine (Aldrich Chemical Co., Milwaukee, Wis.), made $100 \mu \mathrm{g} / \mathrm{ml}$ in distilled water containing $1 \%$ methanol and $0.003 \% \mathrm{H}_{2} \mathrm{O}_{2}$ was added to

\footnotetext{
${ }^{4}$ Hosea, S. W., C. G. Burch, E. J. Brown, R. A. Berg, and M. M. Frank. Impaired immune response of splenectomized patients to the polyvalent pneumococcal vaccine. Submitted for publication.
}

each well and incubated overnight. Thereafter, $5 \mu$ l of concentrated $\mathrm{H}_{2} \mathrm{SO}_{4}$ was added to each well and the color developed in each well was read at $488 \mathrm{~nm}$. The color developed was compared to wells in which PBS-Tween had been substituted for serum during the test procedure. The titer of a serum was the dilution that gave $\geqq 0.05$ OD above control wells. All preimmunization anti-polysaccharide antibody titers were $<1: 20$, and all postimmunization titers were $\geqq 1: 80$. Thus, every animal showed at least a fourfold rise in titer after immunization. There was no difference in postimmunization titer between spx-gp and nl-gp. There was no difference in postimmunization titer, nor in rate of clearance of bacteremia, between spx-gp immunized before or after splenectomy.

In vivo complement depletion. Cobra venom factor (CVF), partially purified venom of Naja naja, was obtained from Cordis Laboratories, Miami, Fla. Nl-gp were injected with CVF (200 U/kg intravenously) 16-20 h before an experiment. With this regimen hemolytic complement C3 titers as measured by a standard titration procedure (18) were $0.1-1 \%$ of normal at the time of bacteremia induction.

Preopsonization. To allow complement to be activated on the surface of the $\mathrm{Pn}$ in the absence of specific antibody, $10^{9}$ Pn were incubated in $15 \mathrm{ml}$ of nonimmune guinea pig serum for $30 \mathrm{~min}$ at $37^{\circ} \mathrm{C}$, washed once in cold PBS, pH 7.4, and resuspended to an appropriate concentration for in vivo experiments. In some experiments the opsonic capability of serum from nonimmune spx-gp was compared to that of nl-gp by incubation of $10^{9} \mathrm{Pn}$ in either spx-gp or nl-gp serum as above.

In vivo and in vitro complement activation by pneumococci. Aliquots of blood drawn from nl-gp immediately before and at timed intervals after the induction of bacteremia were allowed to clot for $1 \mathrm{~h}$ in glass tubes. The serum was removed and stored at $-70^{\circ} \mathrm{C}$ until hemolytic $\mathrm{C} 3$ titers were measured on all samples as an index of in vivo complement activation.

In vitro studies were performed by the addition of varying numbers of washed, heat-killed Pn in $0.5 \mathrm{ml}$ PBS to an equal volume of serum from nonimmune nonbacteremic guinea pigs. The mixtures were incubated in duplicate at $37^{\circ} \mathrm{C}$ for $1 \mathrm{~h}$, and the bacteria were removed by centrifugation. The $\mathrm{C} 3$ titers of such sera after incubation were compared to aliquots of serum incubated under identical conditions with PBS containing no Pn.

Statistical methods. Lethality data were compared using $\chi^{2}$ analysis of survival after challenge with $10^{8}$ organisms. Hepatic and splenic localization and geometric means of blood colony count data were compared using a two-tailed Student's $t$ test.

\section{RESULTS}

Lethality studies. Previous dose-response experiments demonstrated that the $\mathrm{LD}_{50}$ in nl-gp for Ty 12 was about $10^{8} \mathrm{Pn}$ and for Ty $7,>10^{9}$. For comparison of $\mathrm{Ty}$ 12 with Ty 7 in these studies, a single dose of $10^{8}$ organisms was chosen. First, the lethality of Ty 7 and Ty $12 \mathrm{Pn}$ after intravenous injection of $10^{8}$ organisms was compared in nl-gp and spx-gp. As expected from the dose-repsonse experiments, Ty $12 \mathrm{Pn}$ were significantly more lethal than were Ty 7 in $\mathrm{nl}-\mathrm{gp}(P<0.05)$. When these experiments were repeated in spx-gp, it was found that there was a statistically significant increase in mortality of Ty 12 bacteremia after splenectomy $(P<0.05)$, but not of the less virulent Ty 7 infection (Table I). 
TABLE I

Comparison of Survival in Normal and Splenectomized Guinea Pigs

\begin{tabular}{lrrrrrr}
\hline & \multicolumn{2}{c}{ Ty 7} & & \multicolumn{2}{c}{ Ty 12 } \\
\cline { 2 - 3 } \cline { 5 - 6 } & Survived & Died & & Survived & Died \\
\hline Normal & 13 & 1 & & 8 & 9 \\
Splenectomy & 8 & 2 & & 1 & 13 \\
\hline
\end{tabular}

The survival in guinea pigs with or without spleens, injected with $10^{8}$ Pn either Ty 7 or Ty 12. The survival of nl-gp injected with Ty 7 was greater than those injected with Ty 12 $\left(x^{2}=5.42, P<0.05\right)$. The increased survival of spx-gp challenged with Ty 7 compared with Ty 12 was even more marked $\left(\chi^{2}=11.00, P<0.001\right)$. Whereas there was no difference in the survival of spx-gp compared with nl-gp challenged with Ty $7\left(\chi^{2}=0.10, P>0.5\right)$, there was a significant decrease in the survival of spx-gp compared with nl-gp challenged with Ty $12\left(\chi^{2}=4.16, P<0.05\right)$.

Clearance and localization of pneumococci in normal animals. The rate of removal of the two types of Pn from the blood after intravenous injection was next studied using a quantitative colony counting technique. Despite the difference in lethality between the two types of $\mathrm{Pn}$, there was no difference at all in the initial clearance rates (Fig. 1). The distribution of $\mathrm{Pn}$ in the splanchnic reticuloendothelial organs was compared for Ty 7 and Ty 12 during this period of identical clearance. Counts per minute recovered in liver and spleen accounted for $60-70 \%$ of the counts per minute injected, and total splanchnic RES recovery was not dif-

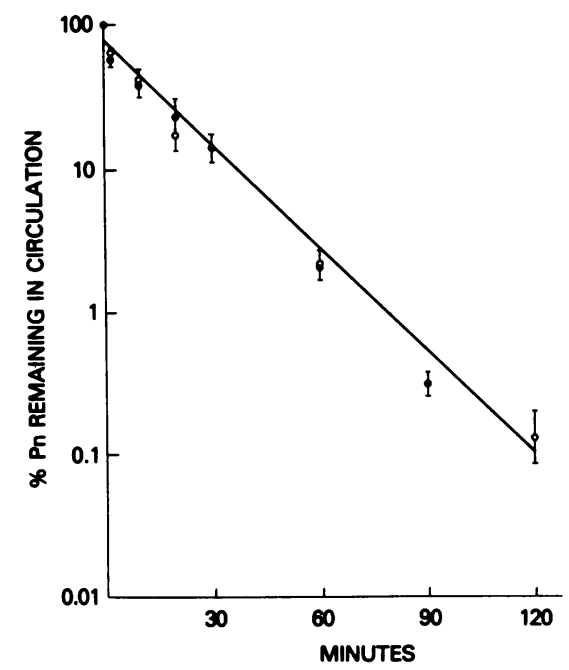

FIgURE 1 The clearance of Ty $7(\odot)$ and Ty $12(O)$ compared during the initial exponential clearance phase. The $\mathrm{Pn}$ remaining in the blood are plotted vs. time, assuming a blood volume equal to $7 \%$ of the body weight for each guinea pig. The clearance rates for the two organisms were identical during this period. ferent between Ty 7 and Ty 12 . An additional 5-10\% of the counts were recoverable in the lung, $5-10 \%$ in the bone marrow, and $<5 \%$ in the blood. The remaining $5-25 \%$ could be recovered in kidney, bladder, muscle, skin, and intestine without specific sites of concentration. The ratio of hepatic to splenic counts for Ty 7 was $5.1 \pm 0.67(n=17)$, whereas for Ty 12 it was $2.7 \pm 0.28(n=15)$. Thus, although the rate of blood stream clearance was identical for the two organisms, the difference in liver to spleen ratio between the two types of Pn represents a significantly greater dependence on splenic clearance for Ty 12 than Ty 7 bacteremia $(P<0.05)$.

Clearance in splenectomized guinea pigs. The kinetics of clearance of Ty $7 \mathrm{Pn}$ in normal and spx-gp are compared in Fig. 2. Clearance is slower in spx-gp than nl-gp, since there are significantly more $\mathrm{Pn}$ in the blood of the spx-gp than the nl-gp at each point. This difference was statistically significant at $P<0.001$ as early as $1 \mathrm{~h}$ after injection. The clearance of Ty 12 in nl-gp and spx-gp is shown in Fig. 3. With Ty $12 \mathrm{Pn}$, clearance is also delayed in spx-gp. Indeed, the difference in clearance between nl-gp and spx-gp is even more marked for Ty 12 than for Ty 7. This, taken together with the previous finding that a greater proportion of RES clearance of bacteria in normal animals occurred via splenic sequestration for Ty 12 than Ty 7, suggested that the extent of splenic clearance in the normal animal was correlated with the magnitude of the defect in clearance of spx-gp. To test this hypothesis, we conducted two further sets of experiments. In the first set, the localization and clearance of a totally nonpathogenic pneumococcus was investigated. In the

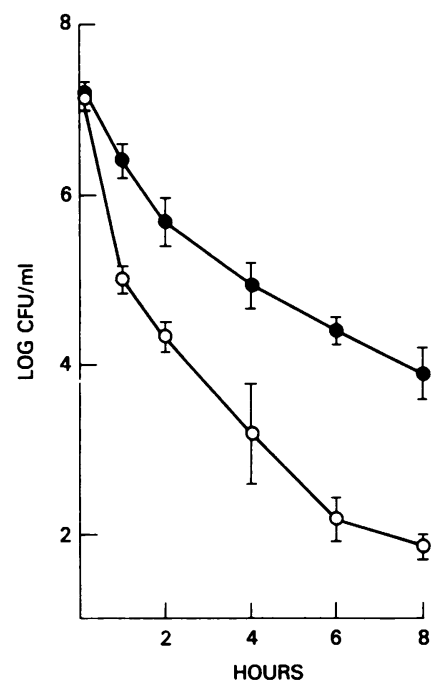

Figure 2 Clearance of Ty $7 \mathrm{Pn}$. The Pn remaining in the blood are plotted vs. time for Ty 7 in nl-gp (O) and spx-gp (O). The rate of clearance is slower for spx-gp than nl-gp. CFU, colony-forming unit. 


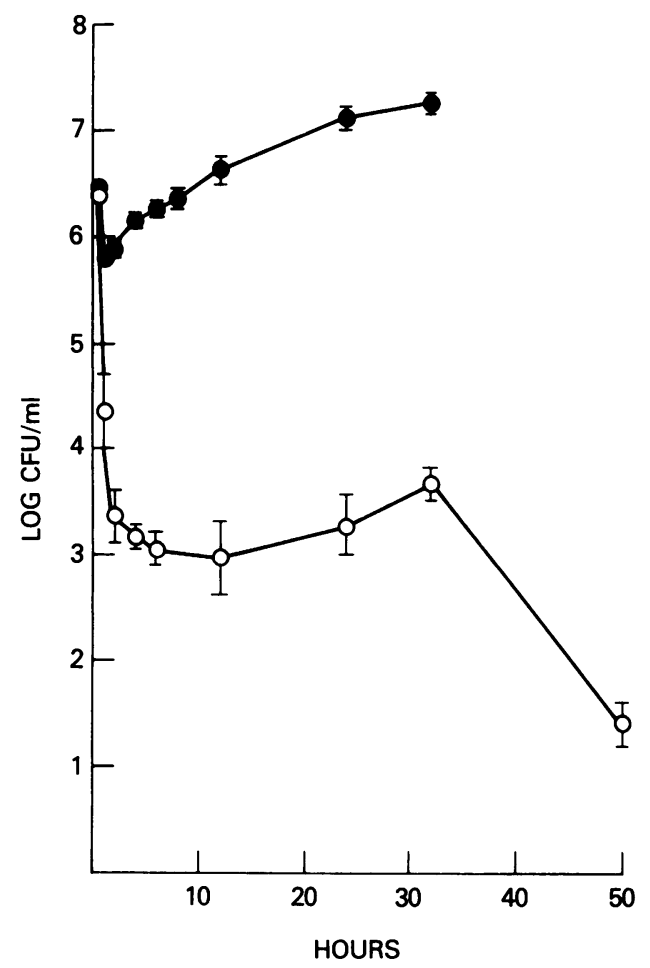

Figure 3 Clearance of Ty $12 \mathrm{Pn}$. The Pn remaining in the blood are plotted vs. time for Ty 12 in nl-gp (O) and spx-gp (O). The rate of clearance is slower for spx-gp than nl-gp, and the difference is more marked than for Ty $7 \mathrm{Pn}$. CFU, colonyforming unit.

second set of experiments, the effects of maneuvers known to increase hepatic sequestration of Pn were examined for their ability to alter blood stream clearance in spx-gp.

Clearance and localization of rough pneumococci. To further investigate the relationship between virulence, RES localization, and the clearance defect induced by splenectomy, unencapsulated (rough) Pn were studied. These organisms are known to be avirulent because they lack a polysaccharide capsule (19). The liver to spleen ratio of pneumococcal clearance 45 min after injection was $9.0 \pm 0.60(n=6)$. There was almost identical blood stream clearance of these organisms in spx-gp and nl-gp (Fig. 4), indicating little, if any, essential role for the spleen in protection against these rough Pn. Hence, these organisms follow the pattern established in the studies of Ty 7 and Ty 12: virulence for both nl-gp and spx-gp correlates with the extent of splenic clearance in nl-gp, as does the extent of the blood stream clearance defect induced by splenectomy.

Effect of immunization and preopsonization. Both immunization of guinea pigs and preopsonization of Pn have previously been shown to increase the extent of hepatic clearance in the normal animals. ${ }^{3}$ It was

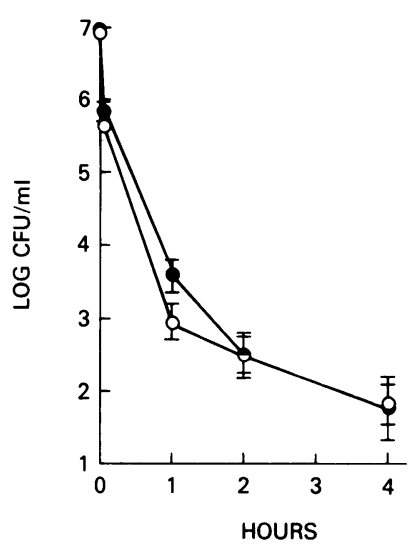

Figure 4 Clearance of rough Pn. The Pn remaining in the blood are plotted vs. time for rough $\mathrm{Pn}$ in $\mathrm{nl}$-gp $(O)$ and spx-gp (O). There is no difference in the rates of clearance of $\mathrm{nl}-\mathrm{gp}$ and spx-gp. CFU, colony-forming unit.

therefore important to determine whether these procedures would improve the blood stream clearance in spx-gp. Immunization, either before or after splenectomy, corrected the clearance defect induced by splenectomy for both Ty 7 and Ty 12 Pn (Fig. 5). Preopsonization also initially corrected the clearance defect. Thus, clearance was as markedly delayed in spx-gp injected with organisms preopsonized in heat-inactivated or EDTA-treated serum as in spx-gp challenged with unopsonized organisms. However, preopsonization with normal nonimmune serum corrected the clearance of spx-gp for $4 \mathrm{~h}$ (Fig. 6). Interestingly, after $4 \mathrm{~h}$ the level of bacteremia in spx-gp injected with opsonized organisms was the same as that in spx-gp injected with unopsonized organisms, organisms preincubated in heat-inactivated serum, or organisms incu-
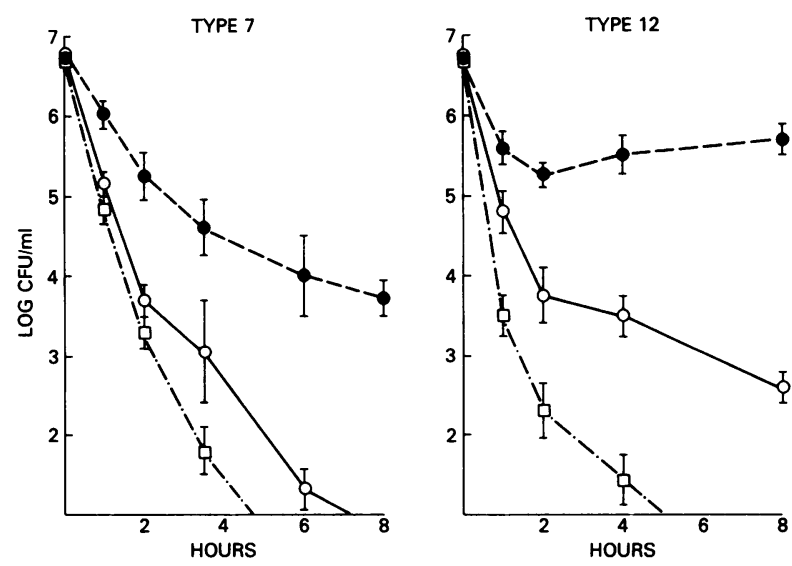

FIgURE 5 Clearance of Ty 7 and Ty 12 in immunized spx-gp. The Pn remaining in the blood are plotted vs. time in unimmunized nl-gp $(O)$ and spx-gp (O), and in immunized spx-gp (口). Immunization corrects the clearance defect of spx-gp for both Ty 7 and Ty 12. CFU-colony-forming unit. 


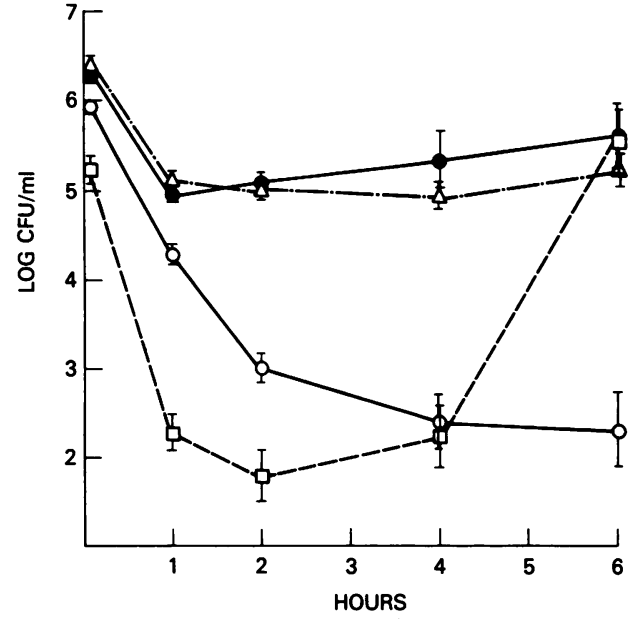

FIgURE 6 The clearance of preopsonized Pn in spx-gp. The Pn remaining in the blood are plotted vs. time for nl-gp (O) and spx-gp (O) given unopsonized Ty 12 , spx-gp given Ty 12 preopsonized in nonimmune guinea pig serum ( $\square$ ), and spx-gp given Ty 12 preopsonized in heat-inactivated guinea pig serum or guinea pig serum made $0.02 \mathrm{M}$ in EDTA $(\Delta)$. Preopsonization with heat labile divalent cation-requiring components in guinea pig serum can correct the clearance defect of spx-gp. CFU, colony-forming unit.

bated in serum made $0.02 \mathrm{M}$ in EDTA. Presumably these live organisms continued to divide after injection and by $4 \mathrm{~h}$ were no longer adequately opsonized for hepatic clearance. Studies of localization of ${ }^{59} \mathrm{Fe}$ labeled Ty $12 \mathrm{Pn}$ confirmed that the improved blood stream clearance resulted from increased hepatic sequestration (Fig. 7). Thus, decreasing dependence on splenic clearance in nl-gp was associated with a disappearance of the blood stream clearance defect of spx-gp.

Splenosis and preopsonization with serum from splenectomized guinea pigs. Two different approaches were used to investigate the possibility that the clearance defect in spx-gp was caused by a serum opsonic defect induced by the splenectomy. In the first set of experiments, intraperitoneal splenosis was deliberately created at the time of splenectomy by implantation of fragments of autologous spleen. Animals treated in this manner were proven to have multiple islands of splenic tissue studding the omentum, bowel wall, and peritoneum by direct visualization as well as by microscopic examination at autopsy 4-8 wk after surgery. Theoretically, the serum of these animals should contain all of the products of splenic synthesis, but an anatomically normal spleen with its normal blood supply is missing. When challenged with (Ty 12) Pn, animals with marked splenosis demonstrated no improvement in the clearance defect seen in spx-gp without splenosis (Fig. 8).

It has been suggested that blood from splenectomized patients may be defective in opsonin (20). Therefore, in a second series of experiments, the ef-

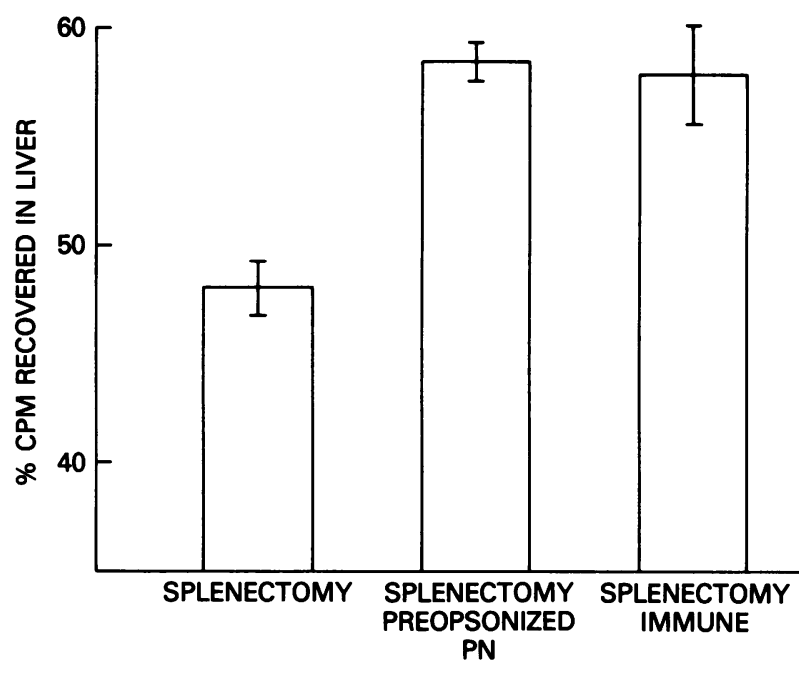

FIgURE 7 Recovery of ${ }^{59} \mathrm{Fe}$-labeled Ty 12 in the liver of spxgp. Pn biologically labeled with ${ }^{59} \mathrm{Fe}$ were injected into spx-gp and hepatic radioactivity assayed $45 \mathrm{~min}$ later. Spx-gp given preopsonized organisms or previously immunized were able to sequester more $\mathrm{Pn}$ in their liver than nonimmune spx-gp given unopsonized organisms, or organisms opsonized with heat-inactivated serum $(P<0.05)$.

fects of preopsonization of Pn in serum from nl-gp and spx-gp on blood stream clearance were compared. In one experiment, the clearance of organisms (Ty 12) preopsonized in serum from spx-gp was compared with that of organisms preopsonized in serum from sham splenectomized guinea pigs when both were injected into spx-gp. Increases in clearance rate, induced by preopsonization in the two sera, were the same. Because heat inactivation and EDTA treatment abolished the

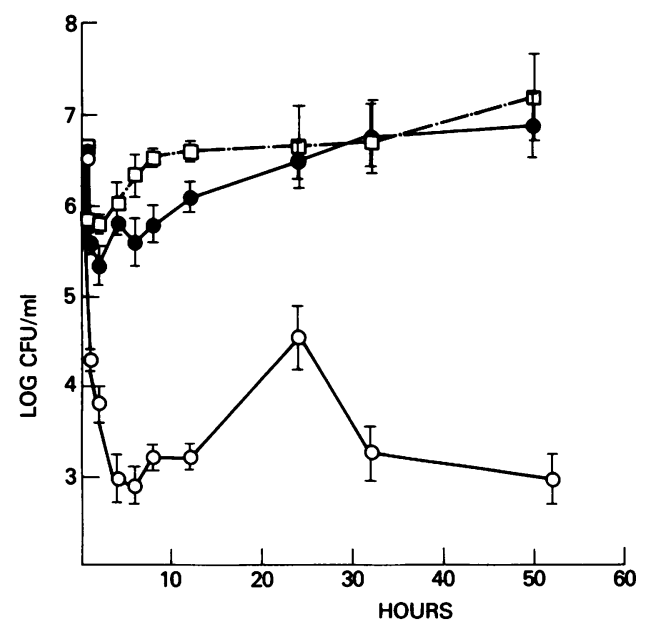

Figure 8 The effect of splenosis on clearance of Ty 12 . The Pn remaining in the blood are plotted vs. time for nl-gp (O), spx-gp (O), and spx-gp in which intraperitoneal splenosis had been deliberately created ( $\square$ ). Splenosis had no effect on the clearance defect of spx-gp. CFU, colony-forming unit. 
capability of preopsonization to augment clearance in spx-gp (Fig. 6), complement activation on the Pn during preopsonization was thought to be the most likely cause of the increased initial clearance observed. Therefore, spx-gp serum and nl-gp serum were compared in their ability to opsonize Ty 12 for clearance in complementdepleted guinea pigs (CVF-GP). If there were a marked defect in complement activation in the serum of spx-gp, they might not have opsonized the $\mathrm{Pn}$ as well as normal serum. Thus, Pn opsonized in spx-gp serum might be cleared less efficiently than Pn opsonized by normal serum in CVF-GP, which are highly dependent on preopsonization of the $\mathrm{Pn}$ to achieve normal clearance rates (16). However, the spx-gp serum was as good or better than nl-gp serum in its ability to promote normal clearance of Pn in CVF-GP (Fig. 9). Loss of the effect of preopsonization after about $4 \mathrm{~h}$, and reversion to the clearance curve obtained when unopsonized organisms were injected into CVF-GP, was observed; this was similar to the phenomenon seen when preopsonized organisms were injected into spx-gp (Fig. 6). These experiments did not support the concept that the essential defect in the spx-gp causing deficient blood stream clearance is the lack of a serum opsonic factor normally synthesized by the spleen.

In vivo and in vitro complement activation. Hemolytic C3 titers decreased markedly after the induction of bacteremia in nl-gp reaching a nadir of one-half of initial values 3-6 $\mathrm{h}$ after the induction of bacteremia. There was no difference between the degree or kinetics of C3 depletion induced by Ty $7(n=5)$ and Ty 12

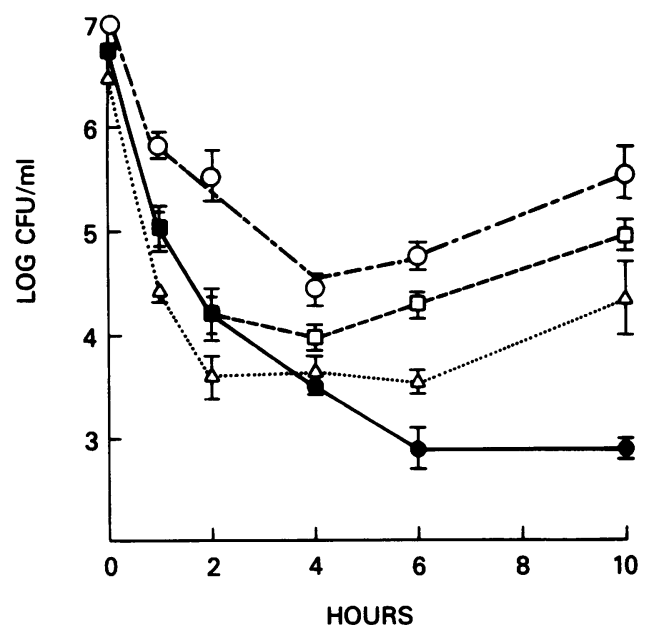

Figure 9 A comparison of the ability of serum from spx-gp and nl-gp to opsonize $P n$. The $P n$ remaining in the blood are plotted vs. time for nl-gp (O), CVF-GP (O), CVF-GP given Ty 12 opsonized with serum from nl-gp ( $\square)$, and CVF-GP given Ty 12 opsonized with serum from spx-gp $(\Delta)$. Nl-gp serum and spx-gp serum are equally able to opsonize Pn for clearance by CVF-GP. CFU, colony-forming unit.
( $n=4)$ Pn. The results of in vitro studies, in which Pn were incubated with nonimmune guinea pig serum, showed a $10 \%$ decrease in C3 titer after incubation with $10^{7} \mathrm{Pn}$, a $50 \%$ decrease in titer upon incubation with $10^{8} \mathrm{Pn}$, and $>90 \%$ decrease after incubation with $10^{9}$ $\mathrm{Pn}$. There was no difference in the degree of $\mathrm{C} 3$ depletion caused by equal numbers of Ty 7 and Ty $12 \mathrm{Pn}$.

\section{DISCUSSION}

These experiments were designed to examine the hypothesis that the division between hepatic and splenic clearance in bacteremia is a key determinant of the outcome of experimental pneumococcal infection. The splanchnic RES distributions of three types of Pn, of varying lethality for guinea pigs, were examined. It was found that the patterns of RES organ localization of the three organisms during initial clearance were significantly different, with the greatest splenic role in clearance demonstrated for the most lethal organism, Ty 12 , and the least for the avirulent rough Pn. This was consistent with our previous observation that conversion of Ty 7 bacteremia from a nonlethal to a lethal infection by depletion of complement was accompanied by a shift in pneumococcal clearance from liver to spleen. ${ }^{2}$ The observation that the most lethal organism is cleared to the greatest extent by the spleen lends further weight to the concept that deficient hepatic clearance may be compensated to some extent by splenic clearance but that this compensation is not complete. ${ }^{2}$

Removal of the spleen would be expected to dramatically alter the course and consequence of bacteremia for an organism that is normally cleared to a significant extent by the spleen, but should have diminished consequences for clearance of organisms less dependent on clearance by the spleen. This was indeed the case for the three types of Pn investigated since splenectomy alters the clearance and lethality of Ty 12 most strikingly, Ty 7 significantly, and rough Pn not at all. The defect of spx-gp in the removal of Ty 7 Pn from the blood implies that there was an important clearance role for the spleen in this infection, despite the lack of increased mortality of this organism in spxgp. It is important to note that a clearance defect and increased mortality induced by splenectomy have been reported for other types of Pn (10-13, 21-23). However, the ability of spx-gp to clear nonpathogenic rough Pn without defect demonstrates that a requirement for splenic sequestration in the blood clearance of $\mathrm{Pn}$ is not simply a property of all Streptococcus pneumoniae. The existence of a clearance defect in spx-gp apparently depends on the presence of a polysaccharide capsule, long recognized as a major pneumococcal virulence factor (24). These experiments with rough and encapsulated Pn provide evidence that, in general, hepatic 
clearance is unable to compensate for the loss of splenic clearance function; only under the ideal circumstances of a nonpathogenic organism is the hepatic clearance function sufficient to sterilize the blood stream normally.

These experiments may be interpreted to mean that it is really the ability of the liver to remove Pn from the blood that determines the extent and consequence of the defect imparted by splenectomy. Because the blood flow to the liver is much greater than to the spleen, there is more opportunity for hepatic than splenic clearance in the normal animal; the spleen serves to remove those organisms bypassed by the hepatic clearance mechanisms. Further support for this concept was obtained from the experiments using immunized guinea pigs or preopsonized Pn. Both these manipulations are known to increase the extent of hepatic localization of Pn in normal animals ${ }^{2}(23,25)$, and would therefore be expected to improve clearance in spx-gp. Indeed, both preopsonization and immunization were able to correct the initial clearance defect of spx-gp and were correlated with increased pneumococcal clearance by the liver. Thus, these maneuvers decreased dependence on the back-up mechanism of splenic sequestration for blood stream clearance. In doing so, they corrected the clearance defect of spx-gp.

Antibody titers were similar in splenectomized animals, whether they were immunized before or after splenectomy, and in normals. Although defective response to intravenously administered antigens has been reported after splenectomy, the defect was overcome by increasing the dose of antigen (26). It may well be that our method of immunization, which is really sublethal infection, represents such a large antigenic load that the defect in antibody synthesis of the splenectomized animals is masked. Moreover, Lozzio and Wargon (27) found that total IgG antibody against sheep erythrocytes in splenectomized mice was not different from normals, and the detecting antibody used in our enzyme-linked immunoabsorbent assay is more sensitive to guinea pig IgG than IgM.

Preopsonization was only able to correct the clearance defect of spx-gp initially, with a return to the clearance curves of unopsonized organisms after 4-8 h. A similar phenomenon was observed when preopsonized organisms were given to complement-deficient guinea pigs (16). Although the explanation of this phenomenon is not entirely clear, it implies at least that the bacteria arising from in vivo division have lost the effects of the in vitro opsonization. It may imply, further, that the $\mathrm{C} 3 \mathrm{~b}$ put on the bacterial surface in vitro is not a sufficient signal for RES macrophage phagocytosis, analogous to conditions demonstrated for the attachment and ingestion of erythrocytes by phagocytic cells in vitro (28).

There have been several reports which imply that the loss of the spleen either surgically or by infarction leads to a serum opsonic defect $(20,29-32)$. This possible explanation for our observations was tentatively rejected for two reasons. First, guinea pigs with extensive splenosis but without a normal spleen were unable to clear Pn any better than spx-gp. Second, serum from the spxgp was comparable to that of nl-gp in its ability to opsonize $\mathrm{Pn}$ for clearance by complement-deficient guinea pigs. In agreement with these findings are experimental data from several other groups $(21,23)$ in which splenosis was unable to protect spx-gp against intravenous challenge with $\mathrm{Pn}$, as well as case reports in humans in whom even extensive splenosis did not protect against the development of overwhelming bacteremia (33). Indeed, the weight of experimental evidence at this point is that although splenosis will decrease the lethality of aerosolized challenge $(22,34)$ it will not protect the animal once sufficient bacteria have reached the blood stream $(21,23)$. Interestingly, models in which partial splenectomy was performed, but the spleen left in its normal relation to its blood supply $(23,35)$ have shown some continued protection of the spleen against overwhelming bacteremia.

Thus it appears that an anatomically normal spleen plays a unique role in the clearance of experimental pneumococcal bacteremia, and that this role is of increasing importance as the pathogenicity of the invading organism increases. When the spleen is removed, the liver is unable to compensate for the loss of its phagocytic activity, and a clearance defect results which is proportional to the importance of the spleen in the clearance of Pn by the normal animal. How the differences in pneumococcal polysaccharide that account for the differences in pneumococcal type cause this variability in rate of removal from the blood stream by guinea pigs is unknown. Complement is an important opsonin in this model (16) and loss of complement has been shown to lead to a shift from hepatic to splenic sequestration (36), so that it is tempting to speculate that differences in complement activation or complement-mediated attachment to RES macrophages may account for the observed differences among the various pneumococcal types. However, we were unable to observe any differences between Ty 7 and Ty 12 in their ability to cause complement depletion either in vivo or in vitro. It is possible that other opsonins, such as fibronectin (37), are involved, or even that metabolic differences among the Pn, such as the rate of division in vivo, are important factors in the way the bacteria interact with the RES. Nonetheless, these experiments indicate a key role for the spleen in the clearance of virulent or poorly opsonized bacteria.

\section{REFERENCES}

1. Enders, J. F., M. F. Shafer, and C. J. Wu. 1936. Studies on natural immunity to pneumococcus type III. III. Correla- 
tions of the behavior in vivo of pneumococci in the certain differences observed in vitro. J. Exp. Med. 64: 307-331.

2. Rogers, D. 1956. Studies on bacteremia. I. Mechanisms relating to the persistence of bacteremia in rabbits following the intravenous injection of staphylococci. J. Exp. Med. 103: 713-746.

3. Martin, S., G. Kerby, and B. C. Holland. 1952. The effect of thorotrast on the removal of bacteria in the splanchnic area of the intact animal. J. Immunol. 68: 293-296.

4. Beeson, P. B., E. S. Brannon, and J. V. Warren. 1945. Observations on the sites of removal of bacteria from the blood in patients with bacterial endocarditis. J. Exp. Med. 81: 9-23.

5. Benacerraf, B., M. M. Sebestyen, and S. Schlossman. 1959. A quantitative study of the kinetics of blood clearance of ${ }^{32}$ P-labelled Escherichia coli and Staphylococci by the reticuloendothelial system. J. Exp. Med. 110: 27-48.

6. Wardlaw, A. C., and J. C. Howard. 1959. A comparative survey of the phagocytosis of different species of bacteria by Kupffer cells. Br. J. Exp. Pathol. 40: 113-117.

7. Evaklis, A. J., S. V. Kevy, L. K. Diamond, and R. E. Gross. 1967. Hazard of overwhelming infection after splenectomy in childhood. N. Engl. J. Med. 276: 1225-1229.

8. Singer, D. B. 1973. Postsplenectomy sepsis. Perspect. Pediatr. Pathol. 1: 285-311.

9. Bisno, A. L., and J. C. Freeman. 1970. The syndrome of asplenia, pneumococcal sepsis, and disseminated intravascular coagulation. Ann. Intern. Med. 72: 389-393.

10. Shinefield, H. R., C. R. Steinberg, and D. Kay. 1966. Effect of splenectomy on the susceptibility of mice inoculated with Diplococcus pneumoniae. J. Exp. Med. 123: 777-794.

11. Bogart, D., W. D. Biggar, and R. A. Good. 1972. Impaired intravascular clearance of pneumococcus type-3 following splenectomy. J. Reticuloendothel. Soc. 11: 77-87.

12. Whitaker, A. N. 1968. The effect of previous splenectomy on the course of pneumococcal bacteremia in mice. $J$. Pathol. Bacteriol. 95: 357-376.

13. Leung, L. S. E., G. J. Szal, and R. H. Drachman. 1972 Increased susceptibility of splenectomized rats to infection with Diplococcus pneumoniae.J. Infect. Dis. 126: 507-513.

14. Gopal, V., and A. L. Bisno. 1977. Fulminant pneumococcal infections in "normal" asplenic hosts. Arch. Intern. Med. 137: 1526-1530.

15. Tomasz, A. 1964. Studies on the competence of genetic transformation in Diplococcus pneumoniae using a synthetic medium. Bacteriol. Proc. 64: 29. (Abstr.)

16. Hosea, S. W., E. J. Brown, and M. M. Frank. 1980. The critical role of complement in experimental pneumococcal bacteremia. J. Infect. Dis. In press.

17. Hosea, S. W., E. J. Brown, C. H. Hammer, and M. M. Frank. 1980. The role of complement in the adult respiratory distress syndrome. J. Clin. Invest. 66: 375-382.

18. Gaither, T. A., and M. M. Frank. 1979. Complement. In Clinical Diagnosis and Management by Laboratory Methods. J. B. Henry, editor. W. B. Saunders Co., Philadelphia. 1245-1462.

19. Heffron, R. 1979. Pneumonia with Special Reference to Pneumococcus Lobar Pneumonia. Harvard University Press, Cambridge, Mass. 18-90.

20. Johnston, R. B., Jr., R. B. Polhil, Jr., and K. M. Pruitt. 1978. Kinetic hemolytic assay of the alternative pathway: deficient activity in sera from some hypogammaglobulinemic and splenectomized individuals. In Clinical As- pects of the Complement System. W. Opferkuch, K. Rother, and D. R. Schultz, editors. Georg Thieme Verlag, Stuttgart. 86-94.

21. Schwartz, A. D., J. F. Goldthorn, J. A. Winkelstein, and A. J. Swift. 1978. Lack of protective effect of autotransplanted splenic tissue to pneumococcal challenge. Blood. 51: 475-478.

22. Dickerman, J. D., S. R. Horner, J. A. Coil, and D. W. Gump. 1979. The protective effect of intraperitoneal splenic autotransplants in mice exposed to aerosolized suspension of type III Streptococcus pneumoniae. Blood. 54: $354-358$.

23. Cooney, D. R., J. C. Dearth, S. E. Swanson, M. K. Dewanjee, and R. L. Telander. 1979. Relative merits of partial splenectomy, splenic reimplantation, and immunization in preventing post splenectomy infection. Surgery (St. Louis). 86: 561-569.

24. White, B. 1979. The biology of the Pneumococcus. Harvard University Press, Cambridge, Mass. 196-212.

25. Schulkind, M. L., E. F. Ellis, and R. T. Smith. 1967. Effect of antibody upon clearance of ${ }^{125}$ I-labelled pneumococci by the spleen and liver. Pediatr. Res. 1: 178-184.

26. Rowley, D. A. 1950. Effect of splenectomy on formation of circulating antibody in the adult male albino rat. J. Immunol. 64: 289-295.

27. Lozzio, B. B. and L. B. Wargon. 1974. Immune competence of hereditarily asplenic mice. Immunology. 27: $167-178$.

28. Mantovani, B., M. Rabinovitch, and V. Nussenzweig. 1972. Phagocytosis of immune complexes by macrophages. Different roles of the macrophage receptor sites for complement (C3) and for immunoglobulin (IgG). J. Exp. Med. 135: 780-792.

29. Winkelstein, J. A., and R. H. Drachman. 1968. Deficiency of pneumococcal serum opsonizing activity in sickle cell disease. N. Engl. J. Med. 279: 459-466.

30. Johnston, R. B., S. L. Newman, and A. G. Struth. 1973. An abnormality of the alternate pathway of complement activation in sickle cell disease. N. Engl. J. Med. 288: 803-808.

31. Hand, W. L., and N. L. King. 1978. Serum opsonization of salmonella in sickle cell anemia. Am.J. Med. 64: 388-395.

32. Constantinopoulos, A., V. A. Najjar, and J. W. Smith. 1972. Tuftsin deficiency: a new syndrome with defective phagocytosis. J. Pediatr. 80: 564-572.

33. Rice, H. M., and P. D. James. 1980. Ectopic splenic tissue failed to prevent fatal pneumococcal septicemia after splenectomy for trauma. Lancet. 1: 565-566.

34. Likhite, V. V. 1978. Protection against fulminant sepsis in splenectomized mice by implantation of autochthonous splenic tissue. Exp. Hematol. (Copenhagen). 6: 433-439.

35. VanWyck, D. B., M. H. Witt, C. L. Witte, and K. Kintner. 1980. Unmasking of serious post splenectomy bacterial clearance defect by complement depletion. Clin. Res. 28: 381A. (Abstr.)

36. Spiegelberg, H. L., P. A. Miescher, and B. Benacerraf. 1963. Studies on the role of complement in the immune clearance of Escherichia coli and rat erythrocytes by the reticuloendothelial system in mice. J. Immunol. 90: 751-759.

37. Saba, T. M., F. A. Blumenstock, P. Weber, and J. E. Kaplan. 1978. Physiologic role of cold insoluble globulin in systemic host defense: implications of its characterization as the opsonic $\alpha_{2}$-surface-binding glycoprotein. Ann. N.Y. Acad. Sci. 312: 43-45. 